PREPARED FOR THE U.S. DEPARTMENT OF ENERGY, UNDER CONTRACT DE-AC02-76CH03073

PPPL-3771

PPPL-3771

UC-70

Random Density Inhomogeneities and Focusability of the Output Pulses for Plasma-based Powerful Raman Amplifiers

by

A.A. Solodov, V.M. Malkin, and N.J. Fisch

January 2003

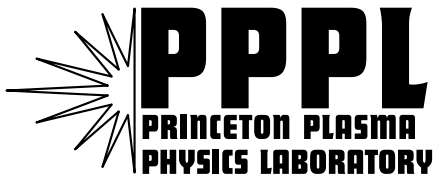

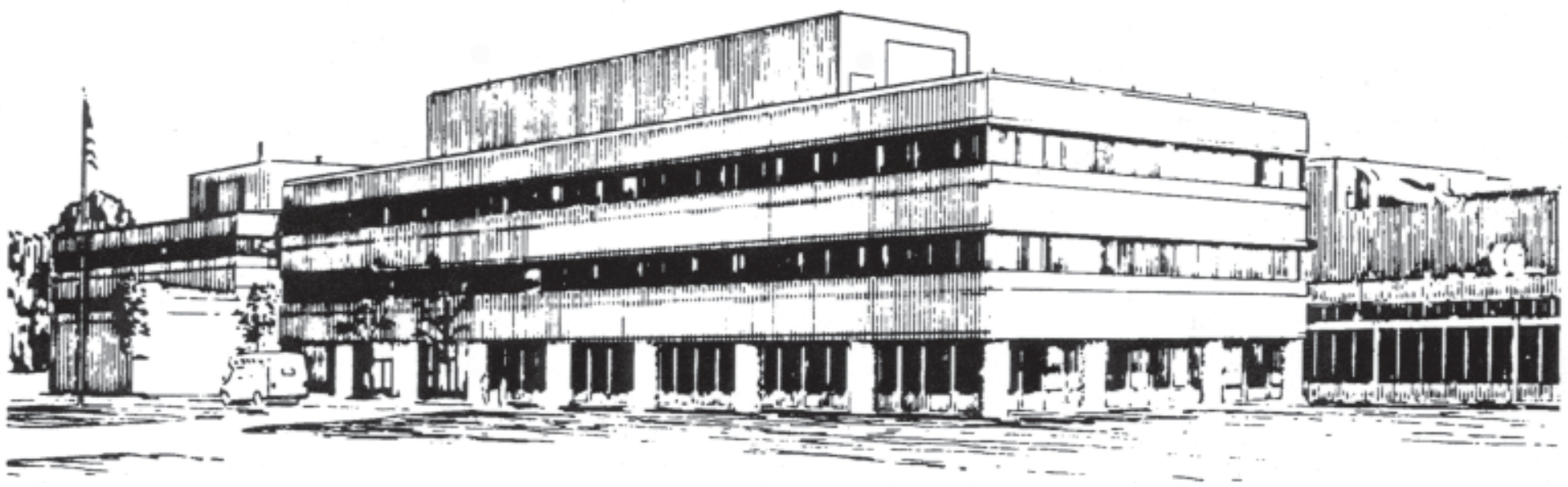

PRINCETON PLASMA PHYSICS LABORATORY PRINCETON UNIVERSITY, PRINCETON, NEW JERSEY 


\section{PPPL Reports Disclaimer}

This report was prepared as an account of work sponsored by an agency of the United States Government. Neither the United States Government nor any agency thereof, nor any of their employees, makes any warranty, express or implied, or assumes any legal liability or responsibility for the accuracy, completeness, or usefulness of any information, apparatus, product, or process disclosed, or represents that its use would not infringe privately owned rights. Reference herein to any specific commercial product, process, or service by trade name, trademark, manufacturer, or otherwise, does not necessarily constitute or imply its endorsement, recommendation, or favoring by the United States Government or any agency thereof. The views and opinions of authors expressed herein do not necessarily state or reflect those of the United States Government or any agency thereof.

\section{Availability}

This report is posted on the U.S. Department of Energy's Princeton Plasma Physics Laboratory Publications and Reports web site in Fiscal Year 2003. The home page for PPPL Reports and Publications is: http://www.pppl.gov/pub_report/

DOE and DOE Contractors can obtain copies of this report from:

U.S. Department of Energy

Office of Scientific and Technical Information

DOE Technical Information Services (DTIS)

P.O. Box 62

Oak Ridge, TN 37831

Telephone: (865) 576-8401

Fax: (865) 576-5728

Email: reports@adonis.osti.gov

This report is available to the general public from:

National Technical Information Service

U.S. Department of Commerce

5285 Port Royal Road

Springfield, VA 22161

Telephone: $1-800-553-6847$ or

(703) $605-6000$

Fax: (703) 321-8547

Internet: http://www.ntis.gov/ordering.htm 


\title{
Random density inhomogeneities and focusability of the output pulses for plasma-based powerful backward Raman amplifiers
}

\author{
A. A. Solodov, V. M. Malkin, N. J. Fisch \\ Princeton Plasma Physics Laboratory, Princeton University, Princeton, New Jersey 08544
}

\begin{abstract}
Random plasma density inhomogeneities may defocus the output pulses of powerful backward Raman amplifiers (BRA). Because of ultra-high intensities of even non-focused BRA outputs, such distortions, if occur, are then difficult to correct. We derive a simple expression for the largest BRA length for which the output pulse focusability is not yet spoiled. Interestingly, this limitation does not depend on the pump laser intensity. We also note a useful effect of plasma inhomogeneities that might help to suppress premature pump backscattering by thermal noise.
\end{abstract}

52.38.-r, 42.25.Dd, 52.35.Mw

\section{INTRODUCTION}

The recently proposed scheme for fast compression of pump laser pulses in plasma through transient backward Raman scattering [1] is expected to produce short laser pulses of nearly relativistic non-focused intensities. For example, for $\lambda=1 \mu \mathrm{m}$-wavelength lasers, the expected non-focused output pulse intensity is $I \sim 10^{17}$ $\mathrm{W} / \mathrm{cm}^{2}$, which is about five orders of magnitude higher than currently available through chirped pulse amplification [2]. Unprecedented ultra-relativistic intensities could be obtained, if the fast compressor outputs were efficiently focused in vacuum. This, however, is not readily achieved by existing mirrors or gratings, since the non-focused output intensities already greatly exceed the highest intensities tolerable by a material surface. The fast compression scheme envisions using a prefocused seed pulse that remains focused as it is amplified in the plasma, with further focusing occurring in a vacuum as the pulse leaves the plasma [1].

The pumped pulses are thought to remain wellfocused through the entire amplification process, because the phase fronts of the original short seed pulse should not be much affected by the pump. The amplification is mediated by the Langmuir wave resonantly excited by beatings of the seed and the pump. It occurs primarily behind the short leading seed, where the Langmuir wave has already time enough to grow; ahead of the seed there are no Langmuir waves, except for small thermal noise. Thus, the growing Langmuir waves backscatter the pump, and the backscattered electromagnetic field also grows behind the seed front. Eventually, the backscattered wave is sufficiently strong to deplete completely the pump. This qualitative picture is confirmed by 2-dimensional numerical simulations [3], where the phase fronts of amplified pulses were shown to be robust to a broad range of pump and seed perturbations in homogeneous plasmas.

The major goal of the current work is to analyze quantitatively the effect of random plasma density inhomogeneities on the focusability of output pulses in backward Raman amplifiers (BRA). Plasma density in- homogeneities can influence the pumped pulse through scattering the seed and through detuning the BRA resonance. The effect on BRA of a fixed longitudinal gradient in plasma density was analyzed and usefully employed in [4]. Here we are interested instead in the effect of random inhomogeneities of the plasma density. The transverse variation of these inhomogeneities is capable of spoiling the focusability of pumped pulses. In the applications that we contemplate, the focusing length of BRA output pulses is assumed to be much larger than the BRA length.

\section{SIMPLE THEORY}

The BRA resonance detuning is caused primarily by fluctuations of the Langmuir wave frequency

$$
\delta \omega_{e} \approx \omega_{0} \delta n / 2 n_{0},
$$

associated with the deviation $\delta n$ of plasma electron concentration from its average value, $n_{0}$, and the respective deviation of electron plasma frequency from its average value, $\omega_{0}=\sqrt{4 \pi n_{0} e^{2} / m_{e}}$, where $e$ is the charge and $m_{e}$ is the mass of an electron. One might expect that the detuning effect is not likely to be important for pumped pulses whose frequency bandwidth is much larger than $\delta \omega_{e}$. The frequency bandwidth of pumped pulses in $\mathrm{BRA}$ is at least as large as growth rate $\gamma_{0}$ of the linear BRA instability of the pump. For a laser frequency much larger the plasma frequency, $\omega \gg \omega_{0}$, the linear growth rate of $\mathrm{BRA}$ is

$$
\gamma_{0} \approx a_{0} \sqrt{\omega \omega_{0} / 2},
$$

where $a_{0}$ is the electron quiver velocity, measured in units of the vacuum speed of light $c$ and normalized such that the pump power density is $I_{0}=$ $\pi c\left(m_{e} c^{2} / e\right)^{2} a_{0}^{2} / \lambda^{2} \approx 2.736 \times 10^{18} a_{0}^{2} / \lambda^{2}[\mu \mathrm{m}] \mathrm{W} / \mathrm{cm}^{2}$. The condition $\delta \omega_{e}<\gamma_{0}$, under which the linear stage of amplification is not affected by plasma density inhomogeneities, takes then the form

$$
\tilde{n} \equiv \delta n / n_{0}<a_{0} \sqrt{2 \omega / \omega_{0}} .
$$


For BRA scheme numerical examples given in the table in Ref. [5], where $a_{0}=0.006$ and $\omega / \omega_{0}=12$, Eq. (3) requires $\tilde{n} \equiv \delta n / n_{0}<0.03$.

This relatively mild condition becomes even milder during the nonlinear stage of amplification, when the pumped pulse becomes shorter and its frequency bandwidth increases. The increase is approximately linear in time, since the pumped pulse duration, which is the duration needed for the pump depletion, is inversely proportional to the pulse amplitude. Taking into account that the major portion of the pump energy is consumed during the advanced nonlinear stage of amplification, when the pumped pulse is the shortest and its bandwidth largest, we do not need in fact to have condition (3) satisfied, but rather a milder condition corresponding to the advanced nonlinear stage of amplification.

When condition (3) is not satisfied, the instability still develops and reaches nonlinear stage in a domain closely trailing the seed, provided the seed is short enough. The length of the instability survival region behind the seed, where the instability is nearly not affected by inhomogeneities, can be estimated as $l_{r}=c / \delta \omega_{e}$. The growth rate there is smaller than $\gamma_{0}$, since $\gamma_{0}$ is the rate that would be reached in a homogeneous plasma much further behind the seed, where the instability is now suppressed by inhomogeneities. In order not to delay too much the pump depletion, one may use more intense seed pulses. The seeding capacity is conveniently characterized, as in Ref. $[1,4,5]$, by the dimensionless integrated amplitude,

$$
\epsilon \approx \sqrt{\omega \omega_{0}} \int b_{0} d t
$$

with the initial seed pulse amplitude $b_{0}$ defined in the same way as the pump amplitude $a_{0}$ above.

Note, that decrease in the linear growth rate at $\delta \omega_{e}>\gamma_{0}$ indicates that density inhomogeneities, apart from having harmful defocusing effect on pumped pulse, might help to stabilize the pump to premature backscattering by thermal noise.

Consider now pumped pulse scattering by plasma density fluctuations, which could spoil the pumped pulse phase fronts and hence focusability. We assume that the leading seed is short enough to be nearly not affected by the detuning and the pump, and also is shorter than the output pumped pulse. According to Ref. [1], the phase does not vary along the pumped pulse in the absence of the detuning. Therefore, when the pumped pulse is already short enough to neglect the detuning within the energy-containing domain, the phase fronts in this domain should closely reproduce the phase fronts of the leading seed. Then, the output focusability depends primarily on the propagation of the seed front, which can be analyzed within the standard linear theory for laser pulse propagation in an inhomogeneous plasma $[6,7]$.
For a single laser pulse propagating through an inhomogeneous plasma in the linear ray optics regime, the wave number deviates by

$$
\delta k \approx-\tilde{n} \frac{\omega_{0}^{2}}{2 \omega c}
$$

from the homogeneous plasma case, resulting in a phase shift

$$
\delta \phi=\int_{0}^{z} d z \delta k \approx-\frac{\omega_{0}^{2}}{2 \omega c} \int_{0}^{z} d z \tilde{n}
$$

at the distance $z$ from the plasma boundary. In particular, this phase shift varies in the transverse directions $\vec{r}_{\perp}$, as does $\tilde{n}$. Thus, an originally quasi-plane section of the pulse phase front becomes distorted in the transverse directions. The $\vec{r}_{\perp}$-dependent part of the laser field can be interpreted as the field scattered by plasma inhomogeneities. As long as $\delta \phi \ll 1$, the $\vec{r}_{\perp}$-dependent intensity constitutes a relatively small fraction $(\delta \phi)^{2}$ of the non-scattered field intensity.

Consider the case that the longitudinal and transverse correlation lengths of plasma density fluctuations satisfy the condition

$$
l_{\|} \ll \pi l_{\perp}^{2} / \lambda=l_{d} .
$$

Then, for an intermediate $z$, such that $l_{\|} \ll z \ll l_{d}$, longitudinal averaging of fluctuations occurs, while diffraction remains negligible and the ray optics approximation can be used. Performing this averaging then gives

$$
\begin{gathered}
(\delta \phi)^{2} \approx\left(\frac{\omega_{0}^{2}}{2 \omega c}\right)^{2} N z, \\
N \equiv<\tilde{n}^{2}>l_{\|} \equiv \\
\int_{-\infty}^{\infty} d Z \tilde{n}\left(\vec{r}_{\perp}, z+Z / 2\right) \tilde{n}\left(\vec{r}_{\perp}, z-Z / 2\right) .
\end{gathered}
$$

The decrease in the non-scattered (i.e., non-distorted) seed field intensity $I_{s}$ is

$$
-\frac{1}{l_{s}} \equiv \frac{d \ln I_{s}}{d z} \approx-\frac{(\delta \phi)^{2}}{z} \approx-\left(\frac{\omega_{0}^{2}}{2 \omega c}\right)^{2} N .
$$

A similar consideration applies to the next $z$-layer and also to the next quasi-planar section of the laser pulse phase front. Therefore, Eq. (10) should be valid everywhere in the plasma. The same arguments will accommodate a slow variation of the correlation function $N$. We do not consider evolution of the scattered field, since it is not needed here, as long as the re-scattering back to the non-scattered field is relatively small.

The quantity $l_{s}$ in Eq. (10) represents the length of energy loss by the well-focused pumped pulse (which can be pumped up to a fluency $2 l_{s} I_{0}$ ). It means that there is not much sense in making the pump longer than $2 l_{s}$ and the plasma should not be longer than $l_{s}$,

$$
z<l_{s} \approx \frac{4 \omega^{2} c^{2}}{\omega_{0}^{4}<\tilde{n}^{2}>l_{\|}} .
$$


This expression does not depend on the pump laser intensity, in contrast to the largest BRA lengths allowed by the near-forward Raman scattering and modulational instability of the pumped pulse (which lengths both get smaller for larger pump intensities $[1,5]$ ).

For instance, $\lambda=1 \mu \mathrm{m}$ and $\omega / \omega_{0}=12$, corresponds to $l_{s} \sim 2 / 10^{5}<\tilde{n}^{2}>l_{\|}$. This $l_{s}$ exceeds the maximum BRA length allowed by the near-forward Raman scattering and modulational instability, $l_{B R A} \sim 0.7 \mathrm{~cm}$ for $a_{0}=0.006$, provided $<\tilde{n}^{2}>l_{\|}<3 \times 10^{-5} \mathrm{~cm}$, i.e. $\tilde{n}<0.03$ for $l_{\|} \sim 0.03 \mathrm{~cm}$.

Alternatively, Eq. (11) could be viewed as a restriction on the amplitude of plasma density fluctuations needed for good output pulse focusability at given amplification length $l_{B R A}$,

$$
<\tilde{n}^{2}>^{1 / 2}<\frac{2 \omega c}{\omega_{0}^{2}\left(l_{B R A} l_{\|}\right)^{1 / 2}} .
$$

\section{NUMERICAL RESULTS}

To verify the above simple theory, we solved numerically the basic equations for three-wave interaction along the 2D set of rays. Neglecting diffraction effects, the basic equations for each ray are 1D equations of Refs. $[1,5]$,

$$
\begin{gathered}
a_{t}+c a_{z}=V b f, \quad b_{t}-c b_{z}=-V a f^{*}, \\
f_{t}+i \delta \omega_{e} f=-V a b^{*}, \quad V=\sqrt{\omega \omega_{e} / 2},
\end{gathered}
$$

where $f$ is a normalized envelope of the Langmuir wave. The shape of the seed was initially Gaussian in both the longitudinal and transverse directions. Further, even when the shape deviates from the Gaussian one, we define the pulse duration and diameter as the respective full widths at the half of the maximum intensity (FWHM).

Random perturbations of plasma density were considered to be Gaussian with the dispersion $\left\langle\tilde{n}^{2}\right\rangle$ and correlation lengths $l_{\|}$and $l_{\perp}$,

$$
\frac{<\tilde{n}(\vec{r}) \tilde{n}(\vec{r}+\vec{R})>}{<\tilde{n}^{2}>}=\exp \left(-\frac{\pi Z^{2}}{l_{\|}^{2}}-\frac{\pi X^{2}}{l_{\perp}^{2}}\right) .
$$

We selected the values of pump amplitude $a_{0}=0.006$, laser-to-plasma frequency ratio $\omega / \omega_{0}=12$, the initial seed amplitude $b_{0}=0.0031$ and duration 40 fs [corresponding to the integrated seed amplitude $\epsilon=0.1$ as defined by Eq. (4)]. The width of the plasma layer was taken $0.7 \mathrm{~cm}$.

Fig. 1 shows the pumped pulse intensity and phase along a single ray, numerically calculated for two different cases of random density perturbations with the same $l_{\|}=0.018 \mathrm{~cm}$ but different amplitudes $\left.<\tilde{n}^{2}\right\rangle^{1 / 2}=$ 0.02 and $<\tilde{n}^{2}>^{1 / 2}=0.15$. The first value of $\tilde{n}$ is smaller, while the second is larger than the "critical" value $<\tilde{n}^{2}>^{1 / 2}=0.03$ that suppresses the linear instability grows rate for the given parameters. For comparison, we show also longitudinal profiles of the pumped pulse intensity in the homogeneous plasma. Note, that the linear $e$-folding length in homogeneous plasma is $l_{0}=c / \gamma_{0}=0.013 \mathrm{~cm}$ for given parameters.
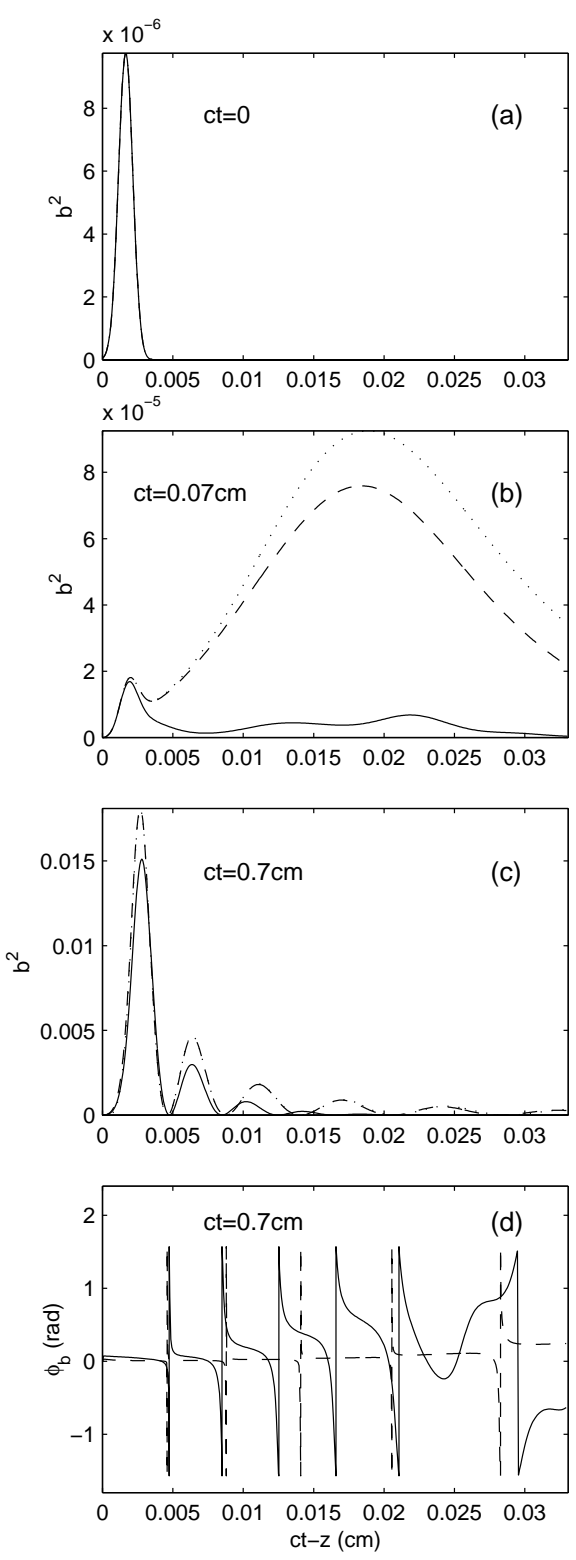

FIG. 1. Longitudinal profiles of the pumped pulse intensity at $t=0(\mathrm{a}), c t=0.07 \mathrm{~cm} \mathrm{(b),} \mathrm{and} c t=0.7 \mathrm{~cm} \mathrm{(c)}$ (dotted, dashed and solid lines correspond to density perturbations of amplitude $\left.<\tilde{n}^{2}\right\rangle^{1 / 2}=0,0.02$, and 0.15 , respectively); the phase profile is given at $c t=0.7 \mathrm{~cm}$ (d).

As seen from the pulse intensity longitudinal profiles at an early amplification stage, $c t=0.07 \mathrm{~cm}$ [Fig. 1 (b)], the linear instability growth rate is not substantially suppressed for $<\tilde{n}^{2}>^{1 / 2}=0.02$ and strongly suppressed for $\left.<\tilde{n}^{2}\right\rangle^{1 / 2}=0.15$ when the instability survives only in a domain closely trailing the seed. 
At the latest stage $c t=0.7 \mathrm{~cm}[$ Fig. 1 (c)], the pulse amplitude profile is much less sensitive to density perturbations: it is nearly the same as in homogeneous plasma for $\left.<\tilde{n}^{2}\right\rangle^{1 / 2}=0.02$ and not too far from that even for $\left.<\tilde{n}^{2}\right\rangle^{1 / 2}=0.15$. This is due to the frequency bandwidth broadening of the pumped pulse, in accordance with the above theory. Thus, highly efficient BRA is capable tolerating very strong density inhomogeneities.

Fig. 1 (d) shows the phase profile of the pumped pulse in inhomogeneous plasma at $c t=0.7 \mathrm{~cm}$. As seen, the phase is nearly constant in the energy-containing domain of the pumped pulse. Phase jumps near zeros of the pulse amplitude are readily understood and not important from the energetic viewpoint. This supports the above speculations that phase fronts of the pumped pulse reproduce phase fronts of the leading seed at the distances shorter than $c / \delta \omega_{e}$ from the front.

Highly efficient BRA along each ray does not guaranty yet good focusability of the output pulse. To address the focusability issue we performed twodimensional simulations including both the amplification of the seed pulse by the pump in plasma and further propagation of the output pulse in vacuum up to the focal plane. The transverse profiles of the input pump and seed intensities were Gaussian with $1.2 \mathrm{~cm}$ FWHM.

The input seed pulse was prefocused to a focal spot with $12 \mu \mathrm{m}$ FWHM, the distance from the plasma boundary to the focal plane was $30.7 \mathrm{~cm}$. The pump and seed on-axis amplitudes, seed duration, and the ratio of the laser frequency to the plasma frequency were taken the same as in Fig. 1. Plasma density perturbations were of the amplitude $\left.<\tilde{n}^{2}\right\rangle^{1 / 2}=0.03$ and the correlation scales $l_{\|}=l_{\perp}=0.02 \mathrm{~cm}$. The results are presented in Fig. 2.

Fig. 2 (a) shows distortion of transverse profiles of the pumped pulse phase in the inhomogeneous plasma. The phase dispersion at the exit from the plasma layer, $<(\delta \phi)^{2}>\approx 0.6$, is consistent with Eq. (8).

Fig. 2 (b) shows the transverse profile of the pumped pulse intensity in the focal plane at $z$-location of the absolute intensity maximum. The intensity is normalized to the maximum focal intensity value in absence of the plasma density inhomogeneities $\left(I_{0}\right)$. The decrease in the non-scattered focused intensity caused by the density inhomogeneities is consistent with Eq. (10), $I_{s} / I_{0}=0.55$. The focal FWHM of the non-scattered spike is the same as in the homogeneous plasma, which is $22 \mu \mathrm{m}$. This size is somewhat larger than it would be in the absence of the pump, due to an "edge steepening" in the transverse profile of the pulse intensity during the amplification. The effect, noted in Ref. [3,4], is associated with the quadratic dependence of pumped pulse amplitude on pump amplitude $\left(b \propto a^{2}\right)$ and horse-shoe shape of the pumped pulse. The "edge steepening" implies generation of larger transverse wave-numbers and, hence, a larger focal spot.

Note that an attempt to make the BRA length larger than $l_{s}$ of Eq. (11), when this is allowed by other limiting factors, would lead to $\left\langle(\delta \phi)^{2}>\gg 1\right.$ and scattering nearly all the pulse energy. We are not interested here in such low-efficiency regimes, even though the nonscattered light still might be seen in the focal spot well above the background. The focal spot remains distinct because the non-scattered field remains well-focused, as the original input, while the scattered light propagates in a broad solid angle. The effect was discussed, for instance, in Ref. [8] in the context of image recognition in a dirty water.

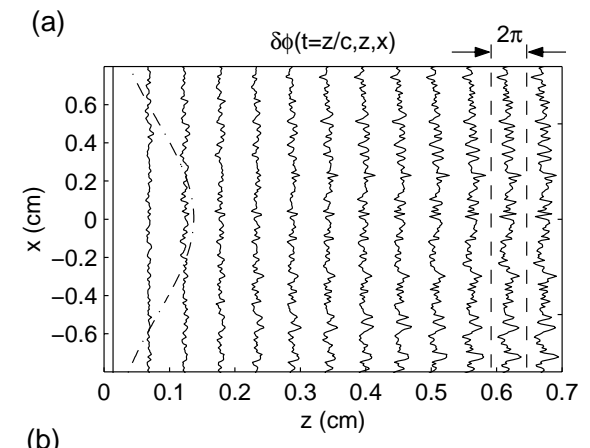

(b)

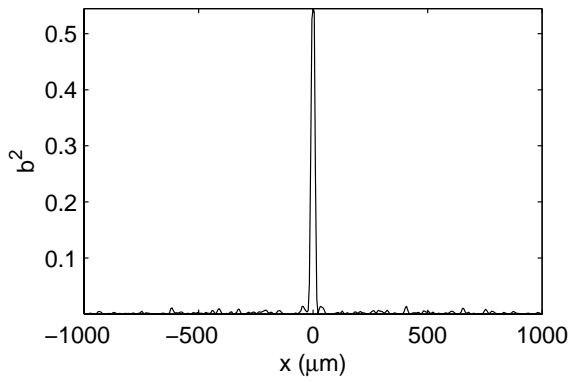

FIG. 2. Transverse profiles of the pumped pulse phase perturbations in the inhomogeneous plasma - solid lines, and the pump intensity (schematic) - dashed-dotted line (a); the transverse profile of the pumped pulse intensity in the focal plane normalized to the maximum focal intensity value in absence of the plasma density perturbations (b).

To illustrate numerically the potentially useful stabilizing effect of plasma density inhomogeneities on the pump backscattering by thermal Langmuir noise, consider propagation of the pump through the plasma with a temperature $T_{e}=40 \mathrm{eV}$. Consider, for simplicity, a thin pump pulse of diameter $d$ much smaller than the amplification length $l$. Then, the waves backscattered at small axial angles $(\theta<d / l)$ might be dangerously amplified, while the waves backscattered at larger angles quickly leave the pump domain in transverse directions. It makes reasonable 1D numerical modeling.

The results of such 1D simulations, performed for $l=0.3 \mathrm{~cm}$ and $d=0.04 \mathrm{~cm}$, are presented in Fig. 3 . The pump amplitude, the laser wavelength, and the ratio of the laser frequency to the plasma frequency were the same as in the above examples. We used 1D equa- 
tions (12). The instability was seeded by thermal Langmuir waves, possessing $T_{e}$ energy per each mode in the three-dimensional wave-vector space,

$$
\frac{\overline{\left|E_{l}^{2}\right|}}{16 \pi}=\frac{1}{2} T_{e} \frac{V_{k}}{(2 \pi)^{3}},
$$

where the bar indicates time averaging and $V_{k}$ is number of Langmuir waves responsible for the pump nearbackscattering, per unit volume. Under above conditions, $V_{k} \sim \Delta k_{\|} \Delta k_{\perp}^{2}$, where $\Delta k_{\|} \sim \gamma_{0} / c$ and $\Delta k_{\perp} \sim$ $k_{\text {pump }} d / l$ (and all these Langmuir waves have wavevectors around $\vec{k} \approx 2 \vec{k}_{\text {pump }}$ ).
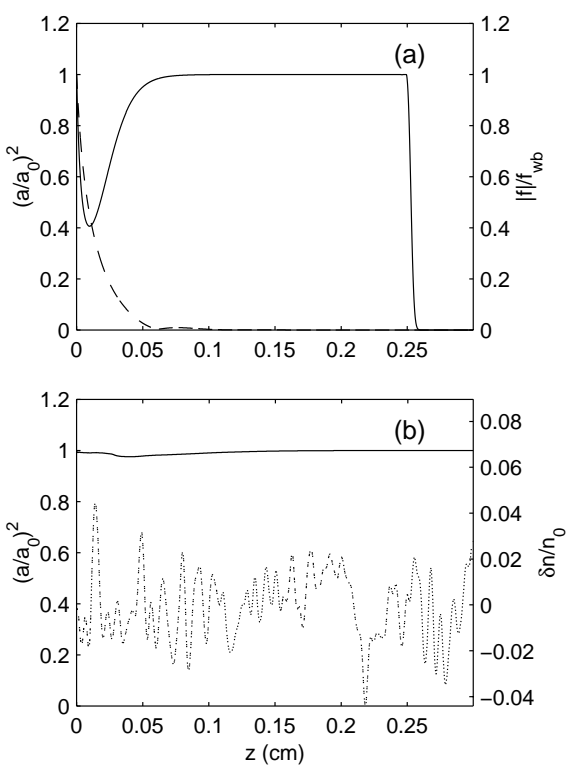

FIG. 3. A pump pulse propagation through a plasma with thermal Langmuir fluctuations: (a) normalized intensities of the pump (solid line) and Langmuir wave (dashed line) at $t=0.85 l / c$ in absence of static density perturbations; (b) normalized pump pulse intensity at $t=2 l / c$ (solid line) and static plasma density perturbations (dotted line).

We compared pump pulse propagation through a homogeneous plasma and through a plasma with static density perturbations of the amplitude $<\tilde{n}^{2}>^{1 / 2}=$ 0.055 and correlation scale $l_{\|}=0.006 \mathrm{~cm}$. In the homogeneous plasma, the pump was noticeably depleted by the noise-seeded backscattering even before the pump front exited the plasma. The pump intensity profile upon the pump front traversing $85 \%$ of plasma length $l$ is shown in Fig. 3 (a) by the solid line. The Langmuir wave amplitude, shown there by the dashed line, reached then the wave-breaking value (see Ref. [1,5]) in the maximum located at the pump entrance into the plasma.

The static plasma density inhomogeneities, shown in Fig. 3 (b) by a dotted line, allows even a $2 l$-long pump pulse (solid line) to traverse the entire $l$-long plasma layer nearly non-depleted.

Note that, in a homogeneous static plasma, the largest depletion occurs near the pump entrance into the plasma where the pumped noise grows the largest time, while in an inhomogeneous static plasma, the major backscattering tends to occur in the longest flat domains of random density profile. To avoid long flat density regions where the plasma is effectively quasihomogeneous, we chose in this simulation the longitudinal correlation scale of static density perturbations smaller than $c / \gamma_{0}$.

\section{CONCLUSION}

Our results indicate that energetically efficient BRA is remarkably robust to the random static plasma density inhomogeneities. Moreover, the output pumped pulse can maintain the same high focusability as the input prefocused seed in a broad range of parameters of practical interest. We also found that random plasma inhomogeneities can help to suppress premature pump backscattering by thermal Langmuir noise.

\section{ACKNOWLEDGMENT}

This work was supported in part by DOE DEFY03098UP00210, DOE AC02-76CH03073, and DARPA.

[1] V. M. Malkin, G. Shvets and N. J. Fisch, Phys. Rev. Lett., 82, 4448 (1999).

[2] G. A. Mourou, C. P. J Barty, and M. D. Perry, Phys. Today, 51, 22 (1998). Perry M.D., D. Pennington, B. C. Stuart, et al., Optics Let., 24, 160 (1999).

[3] G. M. Fraiman, N. A. Yampolsky, V. M. Malkin, and N. J. Fisch, Phys. Plasmas 9, 3205 (2002).

[4] V. M. Malkin, G. Shvets and N. J. Fisch, Phys. Rev. Lett., 84, 1208 (2000).

[5] V. M. Malkin, G. Shvets and N. J. Fisch, Phys. Plasmas 7, 2232 (2000).

[6] A. Ishimaru: Wave propagation and scattering in random media (Academic Press, New York, San Francisco, London, 1978).

[7] S. M. Rytov, Yu. A. Kravtsov, and V. I. Tatarskii: Principles of statistical radiophysics, v. 4 (Springer-Verlag, Berlin, Heidelberg, New York, London, Paris, Tokyo 1988).

[8] A. Ishimaru, Applied Optics 17, 348 (1978). 


\section{External Distribution}

Plasma Research Laboratory, Australian National University, Australia

Professor I.R. Jones, Flinders University, Australia

Professor João Canalle, Instituto de Fisica DEQ/IF - UERJ, Brazil

Mr. Gerson O. Ludwig, Instituto Nacional de Pesquisas, Brazil

Dr. P.H. Sakanaka, Instituto Fisica, Brazil

The Librarian, Culham Laboratory, England

Mrs. S.A. Hutchinson, JET Library, England

Professor M.N. Bussac, Ecole Polytechnique, France

Librarian, Max-Planck-Institut für Plasmaphysik, Germany

Jolan Moldvai, Reports Library, MTA KFKI-ATKI, Hungary

Dr. P. Kaw, Institute for Plasma Research, India

Ms. P.J. Pathak, Librarian, Insitute for Plasma Research, India

Ms. Clelia De Palo, Associazione EURATOM-ENEA, Italy

Dr. G. Grosso, Instituto di Fisica del Plasma, Italy

Librarian, Naka Fusion Research Establishment, JAERI, Japan

Library, Plasma Physics Laboratory, Kyoto University, Japan

Research Information Center, National Institute for Fusion Science, Japan

Dr. O. Mitarai, Kyushu Tokai University, Japan

Library, Academia Sinica, Institute of Plasma Physics, People's Republic of China

Shih-Tung Tsai, Institute of Physics, Chinese Academy of Sciences, People's Republic of China

Dr. S. Mirnov, TRINITI, Troitsk, Russian Federation, Russia

Dr. V.S. Strelkov, Kurchatov Institute, Russian Federation, Russia

Professor Peter Lukac, Katedra Fyziky Plazmy MFF UK, Mlynska dolina F-2, Komenskeho Univerzita, SK-842 15 Bratislava, Slovakia

Dr. G.S. Lee, Korea Basic Science Institute, South Korea

Institute for Plasma Research, University of Maryland, USA

Librarian, Fusion Energy Division, Oak Ridge National Laboratory, USA

Librarian, Institute of Fusion Studies, University of Texas, USA

Librarian, Magnetic Fusion Program, Lawrence Livermore National Laboratory, USA

Library, General Atomics, USA

Plasma Physics Group, Fusion Energy Research Program, University of California at San Diego, USA

Plasma Physics Library, Columbia University, USA

Alkesh Punjabi, Center for Fusion Research and Training, Hampton University, USA

Dr. W.M. Stacey, Fusion Research Center, Georgia Institute of Technology, USA

Dr. John Willis, U.S. Department of Energy, Office of Fusion Energy Sciences, USA

Mr. Paul H. Wright, Indianapolis, Indiana, USA 
The Princeton Plasma Physics Laboratory is operated by Princeton University under contract with the U.S. Department of Energy.

\author{
Information Services \\ Princeton Plasma Physics Laboratory \\ P.O. Box 451 \\ Princeton, NJ 08543
}

Phone: 609-243-2750

Fax: 609-243-2751

e-mail: pppl_info@pppl.gov

Internet Address: http://www.pppl.gov 